Technical note

\title{
Measuring relative positions and orientations of the tibia with respect to the femur using one-channel 3D-tracked A-mode ultrasound tracking system: A cadaveric study
}

\author{
Kenan Niu ${ }^{\mathrm{a}, *}$, Jasper Homminga ${ }^{\mathrm{a}}$, Victor Sluiter ${ }^{\mathrm{a}}$, André Sprengers ${ }^{\mathrm{b}}$, Nico Verdonschot ${ }^{\mathrm{a}, \mathrm{b}}$ \\ a Laboratory of Biomechanical Engineering, Faculty of Engineering Technology, MIRA Institute, University of Twente, Drienerlolaan 5, 7522 NB Enschede, \\ The Netherlands \\ b Orthopaedic Research Lab, Radboud University Medical Center, Nijmegen, The Netherlands
}

\section{A R T I C L E I N F O}

\section{Article history:}

Received 23 June 2017

Revised 11 April 2018

Accepted 30 April 2018

\section{Keywords:}

A-mode ultrasound

Motion tracking

Tibiofemoral kinematics

Non-invasive

Cadaver experiment

Position and orientation

\begin{abstract}
A B S T R A C T
The purpose of this study is to investigate the technical feasibility of measuring relative positions and orientations of the tibia with respect to the femur in an in-vitro experiment by using a 3D-tracked Amode ultrasound system and to determine its accuracy of angular and translational measurements. As A-mode ultrasound is capable of detecting bone surface through soft tissue in a non-invasive manner, the combination of a single A-mode ultrasound transducer with an optical motion tracking system provides the possibility for digitizing the 3D locations of bony points at different anatomical regions on the thigh and the shank. After measuring bony points over a large area of both the femur and tibia, the bone models of the femur and tibia that were segmented from CT or MRI images were registered to the corresponding bony points. Then the relative position of the tibia with respect to the femur could be obtained and the angular and translational components could also be measured. A cadaveric experiment was conducted to assess its accuracy compared to the reference measurement obtained by optical markers fixed to intra-cortical bone pins placed in the femur and tibia. The results showed that the ultrasound system could achieve $0.49 \pm 0.83^{\circ}, 0.85 \pm 1.86^{\circ}$ and $1.85 \pm 2.78^{\circ}$ (mean \pm standard deviation) errors for Flexion-Extension, Adduction-Abduction and External-Internal rotations, respectively, and $-2.22 \pm 3.62 \mathrm{~mm},-2.80 \pm 2.35 \mathrm{~mm}$ and $-1.44 \pm 2.90 \mathrm{~mm}$ errors for Anterior-Posterior, Proximal-Distal and Lateral-Medial translations, respectively. It was concluded that this technique is feasible and facilitates the integration of arrays of A-mode ultrasound transducers with an optical motion tracking system for non-invasive dynamic tibiofemoral kinematics measurement.
\end{abstract}

(c) 2018 IPEM. Published by Elsevier Ltd. All rights reserved.

\section{Introduction}

Detailed knowledge of the skeletal knee kinematics is very important to assess pathologies of the lower limb [1-3]. Accurately measured tibiofemoral kinematics is also useful for evaluation of surgical techniques such as implantation of artificial knee implants $[4,5]$ and for the development and validation of computer models (e.g. musculoskeletal models) capable of simulating normal and pathological human movement [6,7].

Reconstruction of three-dimensional (3D) human movement based on skin-mounted markers has become the standard procedure in clinical human motion analysis [8], where the skinmounted markers are typically taken to represent movement of the

\footnotetext{
* Corresponding author.

E-mail address: niukenan@gmail.com (K. Niu).
}

bony segment beneath the skin. However, the spatial reconstruction of the musculoskeletal system and calculation of its kinematics via a skin marker based multi-link model are subject to Soft Tissue Artifacts (STA) [9]. The markers follow skin movement, but generate errors when used to represent motion of the underlying bony segments.

A wide variety of studies have investigated the quantification and influences of STA in the lower limb during different motor tasks [5,10-21]. These studies found that STA were greater for the thigh than for the shank, with STA errors as high as $50 \mathrm{~mm}$ [7]. In terms of kinematics, an average error of $4.4^{\circ}$ and $13.1^{\circ}$ was found for the three rotation angles and 13.0 and $16.1 \mathrm{~mm}$ for the three translations for walking and running, respectively [11]. In addition, the flexion-extension rotation of the knee joint was found to be determined reliably by skin-mounted markers. However, the remaining motions in the knee joint were more severely affected by STA, which resulted in inaccuracies of relative kinematic outcomes 


\section{One-channel 3D-tracked A-mode ultrasound tracking system}

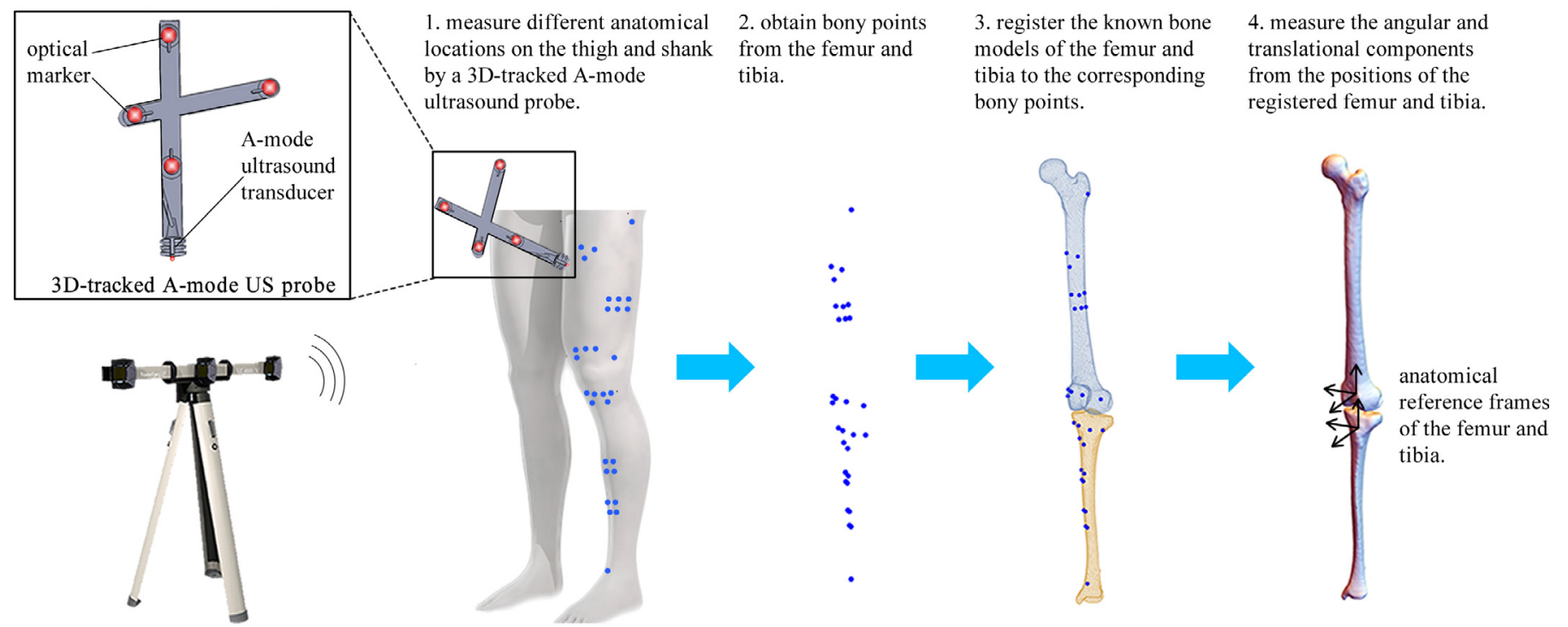

Fig. 1. A schematic of the working principle of the one-channel A-mode ultrasound tracking system.

[12]. To reduce the STA introduced by skin-mounted markers, researchers apply optimization techniques [22,23] and improve the knee model based on advanced joint motion constraints [9]. Although these techniques indeed lead to globally reduced measurement errors of skin markers, the inherent mismatch between skin and bone movement is difficult to remove under all circumstances. Andersen et al. showed that the inclusion of optimized idealized knee joint constraints did not eliminate or reduce the effects of STA and did not improve the validity of the tibiofemoral kinematics derived from skin markers on the thigh and shank [16].

A method to effectively reduce STA is to utilize intra-cortical bone pins rigidly fixed to the bone and equipped with optical markers. This approach has been shown to provide a very accurate estimation of the movement of the femur and tibia in the knee joint $[10,11]$. However, the invasiveness of this method severely limits its in-vivo applicability. Alternatively, fluoroscopic systems have been used to quantify joint motion in vivo [2,14,15,24,25]. Reported accuracies are in the order of $1 \mathrm{~mm}$ and $2^{\circ}$, depending whether a dual or a single fluoroscopic system was used and whether intact knees or implants were involved. In addition to the radiation, a drawback of the fluoroscopic systems is the limited field of view that restricts the patient's natural movement. Recently, fluoroscopic systems that are mobilized by robots which can follow the patient during gait have been proposed, allowing for more natural kinematics $[2,26]$. These types of robotized fluoroscopic systems are, however, still radiative, high in cost and workload. As such it is difficult to implement them in clinical practice on large patient cohorts.

Ultrasound (US) technology is a rapidly developing field with the advantages of non-invasiveness and non-radiation. It has become possible to register US images to the segmented bone in computer-aided orthopedic surgery [27]. The feasibility of estimating knee joint kinematics based on conventional B-mode (Brightness-mode) ultrasound transducers has also been shown [28]. As ultrasound is capable of detecting the bone boundaries through the soft tissue under dynamic motion, the combination of ultrasound technique with a motion tracking system (e.g. optical tracking system) provides a possibility to digitize the detected bone boundaries into 3D bony points. Compared to a conventional
B-mode transducer, an A-mode transducer (i.e. single element ultrasound transducer) is cheaper and smaller in size and more accurate for biometric measurement, e.g. depth $[29,30]$.

Hence, in this study we aimed to demonstrate the feasibility of measuring relative positions and orientations of the tibia with respect to the femur when an ultrasound tracking system was applied in a static fashion. A cadaveric experiment was conducted to assess the accuracy of measured angular and translational measurements compared to reference measurements obtained by optical markers fixed to the intra-cortical bone pins placed in the femur and tibia. Demonstration of this feasibility of accurately measuring the relative positions and orientations of the tibia and femur by one-channel 3D-tracked A-mode ultrasound tracking system in this static study would point towards a level of feasibility of reconstructing tibiofemoral kinematics by combining arrays of A-mode ultrasound transducers with an optical tracking system (i.e. multichannel 3D-tracked A-mode ultrasound tracking system) to quantify tibiofemoral kinematics in dynamic conditions.

\section{Methods}

In this study, we developed a one-channel 3D-tracked A-mode ultrasound tracking system by combining one A-mode ultrasound transducer with optical tracking markers. The 3D-tracked A-mode ultrasound probe was used to measure bony points over a large area of both the femur and the tibia. After this measurement, the known bone models of the femur and the tibia were registered to the corresponding bony points. Then the relative position and orientations of the tibia with respect to the femur was quantified from the position of the registered femur and the registered tibia. The working principle of our proposed system is shown in Fig. 1.

\subsection{The cadaveric experimental setup}

After obtaining ethical approval, one frozen, intact left cadaveric leg (from foot to femoral head) was obtained from the anatomical department of the Radboud University Medical Center (RUNMC). After thawing, two intra-cortical bone pins were screwed into the proximal-anterior part of the femur and the middle shaft of 

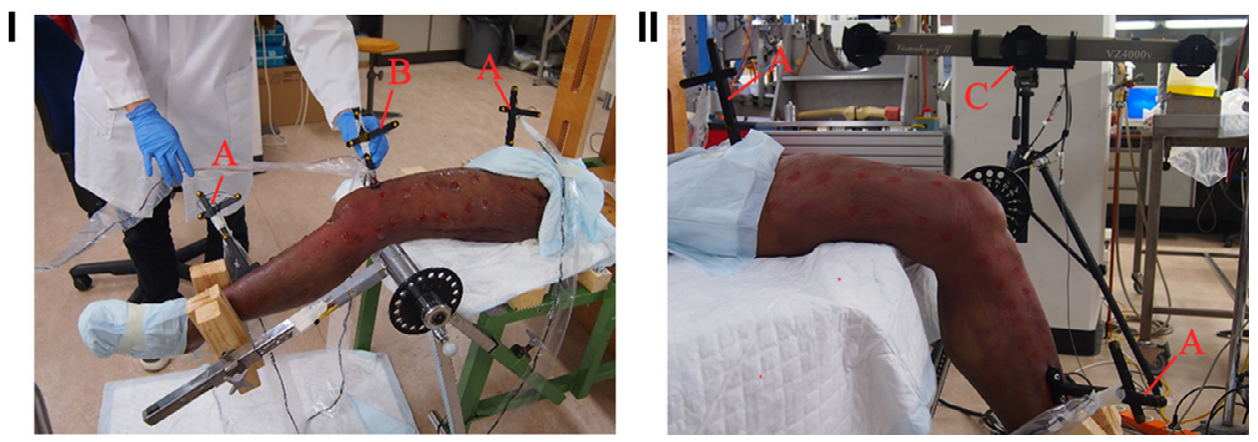

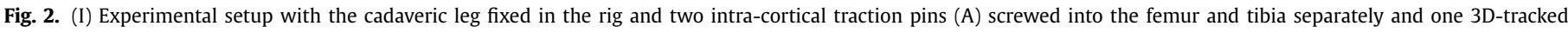

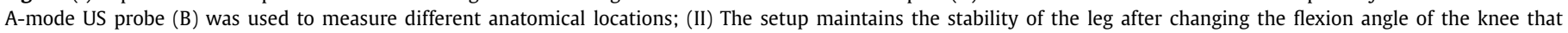
is tracked by Visualeyez tracking system (C).

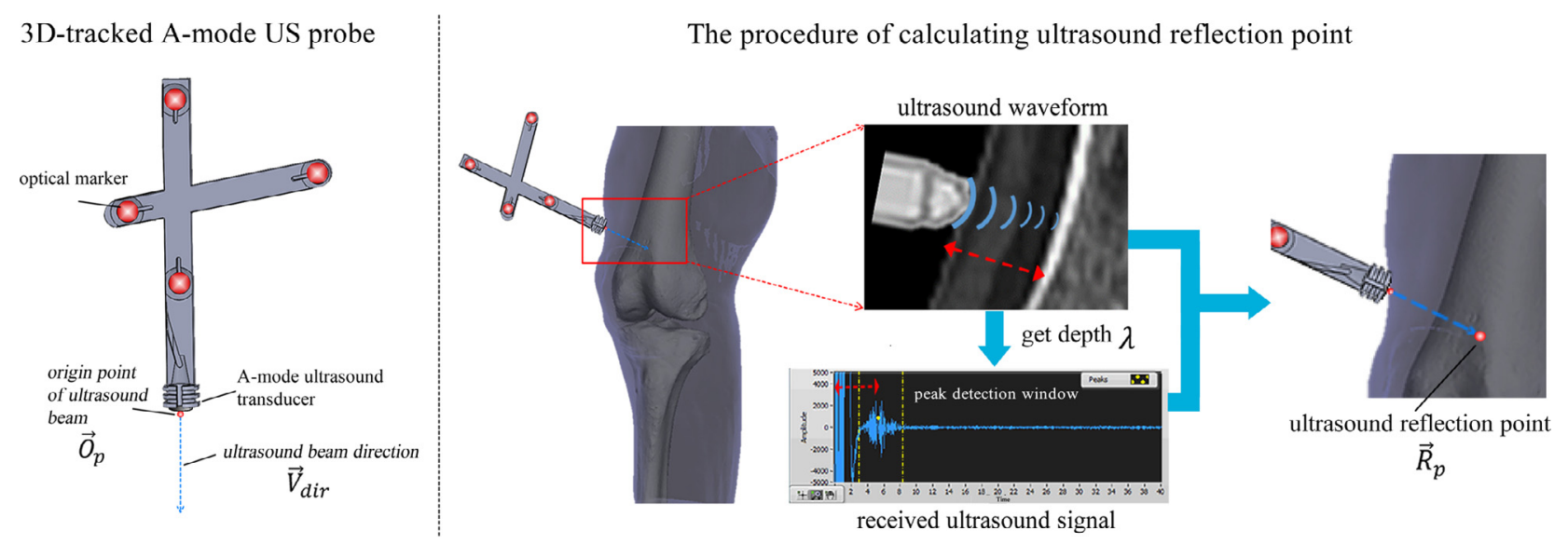

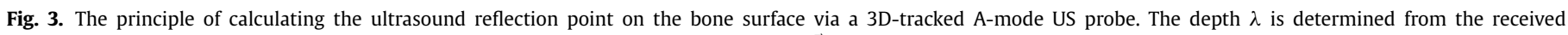

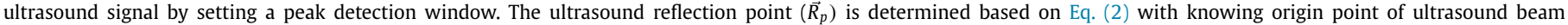
$\left(\vec{O}_{p}\right)$ and unit vector of ultrasound beam direction $\left(\vec{V}_{d i r}\right)$.

the tibia separately, with a rigid structure containing four optical markers that were used to record the reference motions of the bones. After mounting the intra-cortical bone pins, a CT scan was made at the Department of Radiology of the RUNMC using a TOSHIBA Aquilion ONE (TOSHIBA, Tustin, USA) with a voxel size of $0.755 \mathrm{~mm} \times 0.755 \mathrm{~mm} \times 0.500 \mathrm{~mm}$. The $\mathrm{CT}$ images were manually segmented and surface meshes of the femur and tibia in STL format were generated using Mimics ${ }^{\circledR} 17.0$ (Materialise N.V., Leuven, Belgium), including the 3D locations of optical markers of intracortical bone pins. The anatomical reference frames of the femur and tibia were defined from the generated STL Models of the femur and tibia [31]. After CT scanning, the leg was fixated in a flexionextension rig that allowed flexion of the leg in a static manner. The femoral head was rigidly fixed to a pin thereby enabling fixation of the upper leg. The ankle was clamped tightly by wooden blocks to restrict the free movement of the shank (Fig. 2-I). The rig could be manipulated to flex the knee. When the flexion angle of the knee was fixed at a certain angle, the setup guaranteed the stability during ultrasound measurements (Fig. 2-II).

\subsection{D-tracked A-mode US probe}

A single A-mode ultrasound transducer was attached to a custom-made, crucifix-shaped probe containing four optical markers for the tracking system (Fig. 3). The A-mode ultrasound transducer (Imasonic SAS, Voray / l'Ognon, France) had an operating frequency of $7.5 \mathrm{MHz}$ and was focused at $2.5 \mathrm{~cm}$. Two Visualeyez VZ4000v tracking systems (PTI Phoenix Technologies Inc, Vancouver, Canada) were operating at $100 \mathrm{~Hz}$ to measure the trajectories of all optical markers with less than $0.5 \mathrm{~mm}$ RMS error [32]. The ultrasound and Visualeyez systems were integrated in the Diagnostic Sonar FI Toolbox (Diagnostic Sonar Ltd., Livingston, Scotland) based on a National Instruments PXI system (National Instruments, Austin, USA) with $2.3 \mathrm{GHz}$ CPU (Intel Core i7-3610QE) and 8GB RAM. The sample rate of the Diagnostic Sonar system was $40 \mathrm{MHz}$. The acquisition and post-processing software was written in LabVIEW 2014 (National Instruments, Austin, USA).

When probing the 3D-tracked A-mode US probe at different anatomical locations on the thigh and shank, both the received ultrasound signals and the 3D coordinates of the four optical markers were recorded to determine the $3 \mathrm{D}$ coordinates of the ultrasound reflection point (i.e. bony point) on the bone surface (Fig. 3). To get the ultrasound reflection point, firstly the received ultrasound signal was filtered using a second-order low-pass Butterworth filter with a cut-off frequency of $20 \mathrm{MHz}$ and then a peak detection window was set manually in which a detected peak that had greater amplitude than the setting threshold was determined to be the bone surface reflection [3]. To convert the determined peak from time domain to spatial domain in distance unit, the depth $(\lambda)$ from the origin point of ultrasound beam to ultrasound reflection point was calculated using the following equation:

$\lambda=\frac{v t}{2}$

where $v$ is the velocity of sound in the material $(1590 \mathrm{~m} / \mathrm{s}$ in muscle across the fibers) [33] and $t$ represents the time that ultrasound waveform takes from the origin of ultrasound beam to the bone surface and reflects back to the origin. Secondly, the origin point $\left(\vec{O}_{p}\right)$ and the unit pointing direction $\left(\vec{V}_{d i r}\right)$ of the US beam were 
A

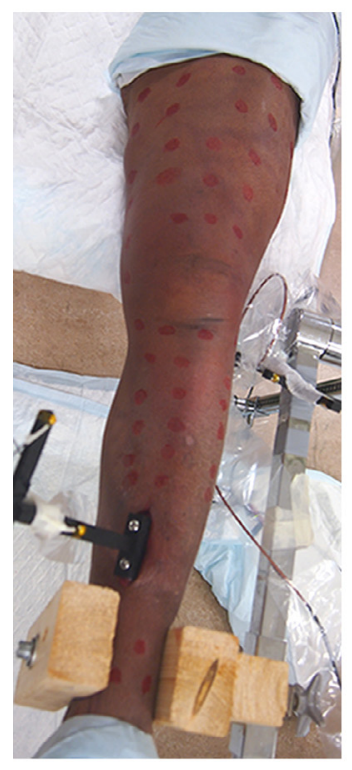

B

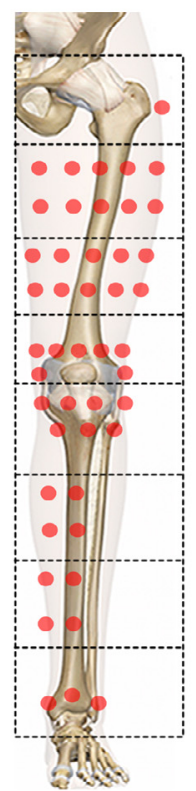

C

great trochanter

femur proximal

femur middle

femur distal

tibia proximial

tibia middle

tibia distal

tibia ankle

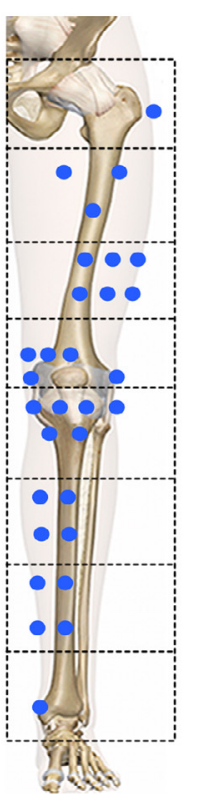

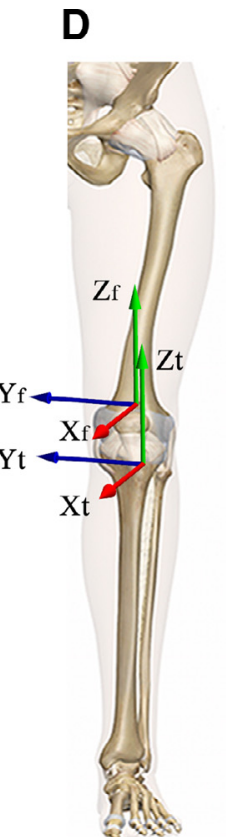

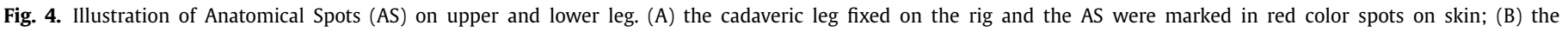

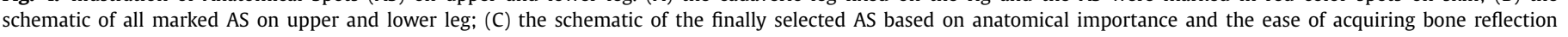
points; (D) the illustration of the femoral and tibial anatomical reference frames.

determined from a calibration method described in [3]. Subsequently, the $3 \mathrm{D}$ coordinates of ultrasound reflection point $\left(\vec{R}_{p}\right)$ were calculated by:

$\vec{R}_{p}=\vec{O}_{p}+\lambda \vec{V}_{d i r}$

where $\vec{O}_{p}$ represents the original 3D coordinates of US beam and $\vec{V}_{\text {dir }}$ represents the unit vector of the direction of US beam and $\lambda$ is the measured depth from Eq. (1). The abovementioned method was used to calculate ultrasound reflection points at different anatomical locations on the femur and tibia.

\subsection{Measurement protocol}

Since only one 3D-tracked A-mode US probe was used in this study, the cadaveric leg needed to be kept in various stationary poses so that we could obtain multiple bony points from the femur and tibia. After collecting all bony points at one stationary pose, the leg was flexed to a new position where a new set of bony points was collected. The cadaveric leg was flexed and fixed at five different positions for ultrasound measurements ranging from full extension to an approximate flexion angle of $90^{\circ}$. In addition, to ensure that the bony points measured at five different knee poses were consistently acquired at the identical anatomical areas, we divided the upper and lower leg into eight regions of interest which contained Anatomical Spots (AS, see Fig. 4) marked with red spots on the skin where we attempted to measure the bony points with the 3D-tracked A-mode US probe. A total of 28 AS were marked on the thigh and 18 AS were marked on the shank (Fig. 4-A, B).

From a practical point of view the number of ultrasound transducers of multi-channel A-mode US tracking system will be limited due to issues with limitations in electronics, cabling and costs, line-of-sight problem of each optical marker. The number of points was determined by a practical situation where the affordability for multiple transducers was limited in the future. Therefore, in this study, we chose to use a total of 30 ultrasound reflection points to enable calculation of the position of the tibia relative to the femur; 15 points from the femur and 15 points from the tibia. As the lateral and medial epicondyles of femur and tibia, greater trochanter and ankle joint are important anatomical landmarks for lower limb intra-operative registration in orthopedic surgery [3,34], we prescribed that those anatomical landmarks were to be included in selected AS. The remaining AS were selected based on the level of ease of acquiring a valid bone reflection from each AS at different flexion angles. The final distribution of the selected AS is shown in Fig. 4-C. When the cadaveric leg was flexed and fixed at one pose, six ultrasound measurement trials were recorded at each selected AS. Each trial was a single shot measurement. Hence, in total, 180 $(6 \times 30)$ bony points were measured for one fixed pose. The duration of capturing 180 bony points was about $1 \mathrm{~h}$

\subsection{Estimation of the relative tibia-femoral position}

After measuring all selected AS for five flexion angles, a dataset including $900(180 \times 5)$ ultrasound reflection points was measured. Each of the trials involved a point cloud consisting of 30 bony points from the femur and tibia. A registration method was used for each trial to register 30 bony point to the femur and tibia. More detailed information can be found in supplementary material. After registration, the relative positions and orientations of the tibia with respect to the femur were quantified using the method described by Grood and Suntay [35]. The measured angular and translation components were compared to the reference measurements which were derived utilizing the 3D locations of intra-cortical bone pins on the femur and tibia using a point-to-point registration algorithm [36] at the different poses. Using the selected AS (Fig. 4-C), the registration algorithm was employed in 6 consecutive trials for every pose ( 5 poses in total).

\section{Results}

For flexion $(+) /$ extension( $(-)$ rotation, the mean \pm standard deviation error were sub-degree $\left(0.49 \pm 0.85^{\circ}\right)$ compared with reference angular measurements (Fig. 5; Table 1). Root-Mean-Square (RMS) errors ranged from $0.95^{\circ}$ to $3.30^{\circ}$ for joint rotations and ranged from $3.20 \mathrm{~mm}$ to $4.20 \mathrm{~mm}$ for joint translations. The largest rotational error was associated with external-internal rotation 

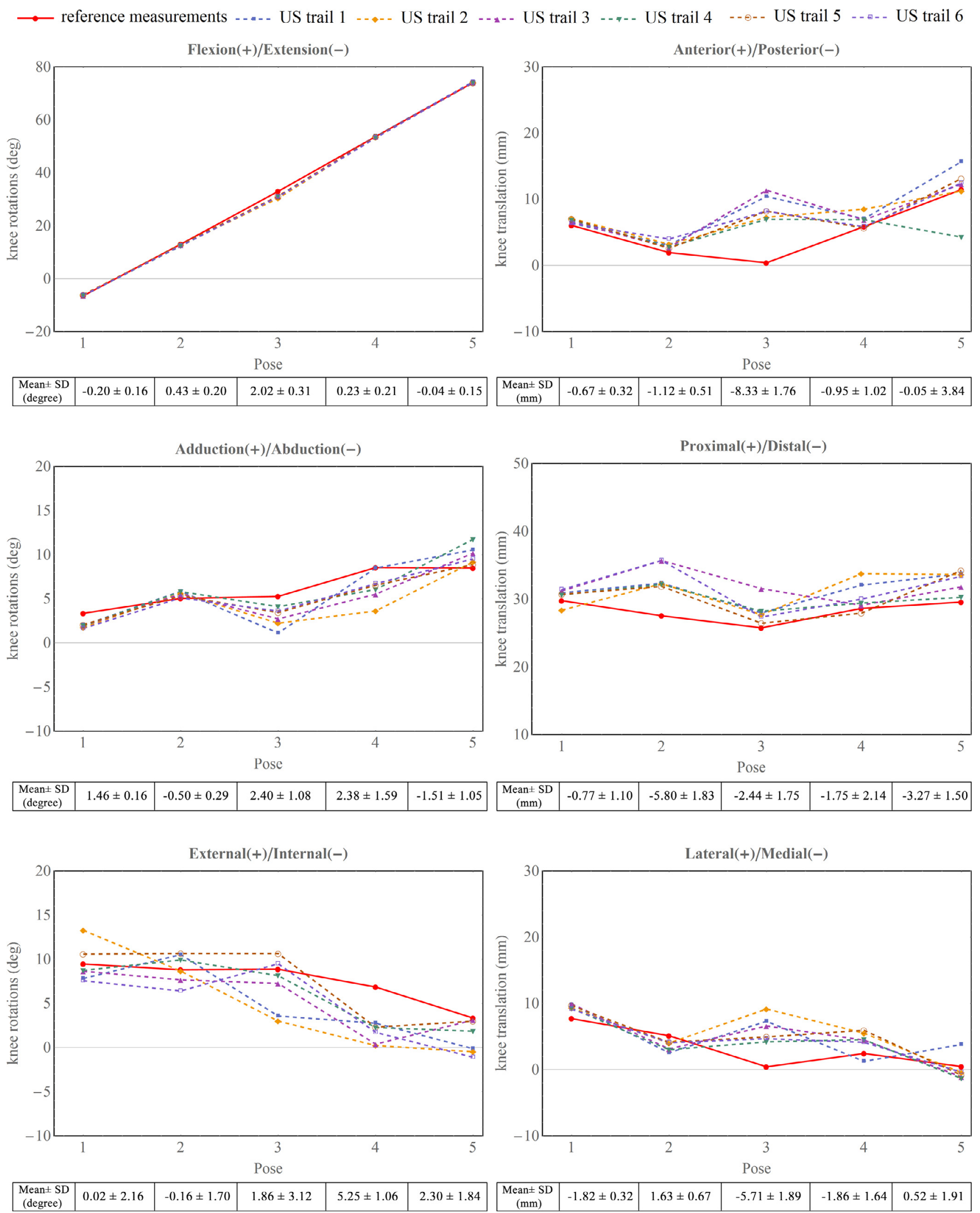

Fig. 5. Comparisons of the six US trials of ultrasound determined angular and translational measurements (dashed lines) and the reference measurement (red, solid line) on knee joint flexion $(+) /$ extension $(-)$, adduction $(+) /$ abduction $(-)$ and external $(+) /$ internal $(-)$ rotations and anterior $(+) /$ posterior $(-)$, proximal $(+) / \operatorname{distal}(-)$ and lateral $(+) /$ medial $(-)$ translations. The tables below each plot represent the mean and standard deviation of the differences between six US measurements and the reference measurement at each pose. 


\section{Table 1}

Mean, Standard Deviation (SD) and Root-Mean-Square (RMS) errors across six US trials for relative angular and translational measurements compared to reference measurement: Flexion-Extension (Flex/Ext), Adduction-Abduction (Add/Abd), External-Internal (Ext/Int) rotations; Anterior-Posterior (Ant/Post), ProximalDistal (Prox/Dist) and Lateral-Medial (Lat/Med) translations.

\begin{tabular}{|c|c|c|c|c|c|c|}
\hline & \multicolumn{3}{|c|}{ Joint rotational errors $\left({ }^{\circ}\right)$} & \multicolumn{3}{|c|}{ Joint translational errors (mm) } \\
\hline & Flex/Ext & Add/Abd & Ext/Int & Ant/Post & Prox/Dist & Lat/Med \\
\hline Mean & 0.49 & 0.85 & 1.85 & -2.22 & -2.80 & -1.44 \\
\hline SD & 0.83 & 1.86 & 2.78 & 3.62 & 2.35 & 2.90 \\
\hline RMS & 0.95 & 2.01 & 3.30 & 4.20 & 3.63 & 3.20 \\
\hline
\end{tabular}

whereas the anterior-posterior translation produced the largest translational error.

\section{Discussion}

This study has shown that a one-channel 3D-tracked A-mode US tracking system can measure relative positions and orientations of the tibia with respect to the femur in a static fashion with an accuracy of $0.95^{\circ}$ to $3.30^{\circ}$ (RMS) for rotations and $3.20 \mathrm{~mm}$ to $4.20 \mathrm{~mm}$ (RMS) for translations. A rather unique feature of this system is the combination of an A-mode ultrasound transducer with a conventional motion tracking system (optical tracking system in this study) to measure the locations of bony points instead of markers mounted on the skin. Subsequently, the registration method was applied to the acquired cloud of bony points to estimate the relative position of the tibia with respect to the femur. In this study we used a cadaver experiment to demonstrate its technical feasibility. With this study the technique has shown the potential to be extended to a multi-channel 3D-tracked A-mode ultrasound tracking system for measuring dynamic movements. Dynamic tibiofemoral kinematic measurements in 3D space require the determination of the instantaneous position and orientation of the femur and tibia [37]. This study demonstrates the feasibility of such system. However, to complete such a system, a number of issues involved in dynamically measuring bony points and estimating the position and orientation of bone under dynamic conditions will be investigated in the future studies.

In contrast to the utilization of skin-mounted marker systems where the measured kinematics associated with skin-mounted marker are subject to STA (i.e. differences between marker and bone positions) and the effects of STA are difficult to eliminate $[37,38]$, we utilized the measured ultrasound reflection points to localize bone surface in order to estimate the position and orientation of underlying bone [3], which has the potential to overcome the effect of STA. Therefore, this technique has the potential to be applied in gait analysis studies. However, in this paper, we did not perform a direct comparison with a skin marker system. To investigate this, a multi-channel 3D-tracked A-mode ultrasound tracking system is necessary for simultaneous ultrasound and skin marker measurements.

Relative to our concept, the utilization of a fluoroscopy system to estimate the position and orientation of bone is different, since it relies on the radiological images and adequate modelbased techniques $[24,39,40]$. Fluoroscopy systems provide highly accurate measurements of tibiofemoral kinematics, especially for knee with implants [26,39]. Guan et al. reported that maximum root-mean-squared errors were $0.33 \mathrm{~mm}$ and $0.65^{\circ}$ for translations and rotations of the TKA knee and $0.78 \mathrm{~mm}$ and $0.77^{\circ}$ for translations and rotations of the intact knee [26]. The maximum rootmean-squared errors were $4.20 \mathrm{~mm}$ and $3.30^{\circ}$ for translations and rotations of the intact knee for our one-channel ultrasound system, which is much less accurate than a biplane fluoroscopy system. However, relative to fluoroscopic systems, the advantages of our system are the larger field of view (similar to conventional skin marker systems) and the lack of radiation.

We expect the accuracies of a multi-channel 3D-tracked Amode ultrasound tracking system to be about $1 \mathrm{~mm}$ and $1^{\circ}$ for translational and rotational errors in the further development, although that will be very challenging when considering several sources of inaccuracies, both technological and human factors. For the one-channel US system as demonstrated in this study, the accuracy of measured angular components could achieve $0.49 \pm 0.83^{\circ}$ $($ Mean \pm SD) errors for flexion-extension. However, there were large errors for adduction-abduction rotation $\left(0.85 \pm 1.86^{\circ}\right)$ and external-internal rotation $\left(1.85 \pm 2.78^{\circ}\right)$ and the maximum translational errors was $-2.22 \pm 3.62 \mathrm{~mm}$ for Anterior-Posterior translation. In its current form the system does not fulfill the requirements yet. Hence, we aim for achieving higher accuracies for the multi-channel ultrasound tracking system in the future.

Considering the working principle of this technique, there are two types of errors caused inaccurate measurements: (1) the extrinsic errors in detection of a bony point; (2) intrinsic errors of registration algorithm. The extrinsic errors in the bony point detection mainly stem from the detection of the wrong peak caused by the problem of distinguishing between real and spurious peaks amongst the noisy ultrasound receiving signal. This process was done manually by placing a detection window in the ultrasound receiving signal. As Fig. 5 shows, the third pose generally had larger errors than rest of poses. Especially in Anterior-Posterior plot, there was $-8.33 \pm 1.76 \mathrm{~mm}$ for the third pose. However, the errors of rest of poses were considerably low. That was the reason why the overall error of anterior-posterior translation became the largest erroneous measurement in translations. To mitigate this type of error, more advanced and robust peak detection methods can be developed. The intrinsic errors were caused by the fact that both the femur and tibia are rather tubular in shape. Hence, a small number of points on the tubular surface do not provide sufficient constraints on external-internal rotation and proximaldistal translation. As the bony points located on the two ends of bone (e.g. condyles of femur, femoral head) are inaccessible for the ultrasound beam, the registration method gives relative weak constraints for external-internal rotation and proximal-distal translation and also is susceptible to be stuck in a local minimum. More robust registration algorithm can be developed to tackle this specific registration problem in the future.

This study has several limitations. Firstly, we used only one cadaver leg in the experiment and more cadaveric specimens could provide more robustness information regarding the differences of geometry of bony segment and the thickness of soft tissue. Secondly, we put the cadaver leg in a rig and moved the leg in different static poses. Therefore, there was no dynamic muscle activation and the soft tissues may have moved differently relative to the bone than under in-vivo conditions. This may influence the expected accuracy of the US signal when used under in-vivo conditions. Future studies will focus on dynamically measuring ultrasound signals and investigating the way to fix the 3D-tracked Amode US probe for different anatomical locations. In future studies special ultrasound holders that are customized for different anatomical regions will be one of the solutions to combine optical markers and arrays of A-mode ultrasound transducers for detecting reliable bony points. Thirdly, no direct comparison with a skin marker system and a fluoroscopic system was included in this study, preventing quantitative comparison between the systems.

Obviously, this study also has strengths. Firstly, it focused on investigating the feasibility of this system. The experiment was conducted in a highly controlled manner with navigated intra-cortical bone pins as ground truth. This allowed for the best way to define the errors of the US system. Secondly, we used a single A-mode US system and applied it to static positions of the knee joint. By 
using this method we found a cheap and fast way to assess the potential of a multi-channel system without actually acquiring all equipment and developing all software integrations.

The results from this study, demonstrating the efficacy of a single A-mode transducer, can be extended to a multiple transducers system that maybe create a new approach of non-invasive measurement of tibiofemoral kinematics in gait analysis and detailed prosthetic measurement. Further research needs to be directed to implementing the multi-channel 3D-tracked A-mode ultrasound tracking system. In-vitro and in-vivo experiments will need to be conducted to validate the multi-channel A-mode US tracking system under dynamic circumstances.

\section{Conclusion}

This study has presented a one-channel 3D-tracked A-mode ultrasound tracking system and proven its feasibility for measuring relative positions and orientations of the tibia with respect to the femur in a cadaveric experiment. Our proposed 3D-tracked A-mode ultrasound system could achieve root mean square errors ranging from $0.95^{\circ}$ to $3.30^{\circ}$ and $3.20 \mathrm{~mm}$ to $4.20 \mathrm{~mm}$ for rotational components and translational components, respectively. Although there are opportunities to further reduce the errors, this study already demonstrates the feasibility of extending it to a multi-channel Amode ultrasound tracking system for measuring tibiofemoral kinematics under dynamic conditions.

\section{Acknowledgments}

The authors also thank for the generous helps of Léon Driessen and Richard van Swam in cadaveric preparation from Orthopedic Research Lab, Radboud university medical center, Netherlands.

\section{Competing Interests}

The authors have declared that no competing interests exist.

\section{Funding}

The research leading to these results has received funding from the European Research Council under the European Union's Seventh Framework Programme (FP/2007-2013) / ERC Grant Agreement n. 323091 awarded to N. Verdonschot.

\section{Ethical approval}

In this study we used one human cadaveric specimen. Testing on cadaveric knees is necessary to ensure the safety and functioning of the developed technology before we apply this to patients. At the Radboud University Medical Center (RUNMC) we have a long history of performing cadaveric experiments and we established working principles to ensure all ethical issues and legal aspects are covered. RUNMC has an Anatomical department which has the authority under Dutch law to use human tissue for educational and research purposes. The Dean of the Medical faculty is responsible for the ethical issues with regard to the use of human cadaver material. The protocol of the experiment was assessed by an authorized person at the anatomical department and approved our protocol; which grants us the ethical approval to perform the study.

\section{Supplementary materials}

Supplementary material associated with this article can be found, in the online version, at doi:10.1016/j.medengphy.2018.04. 015.

\section{References}

[1] Reinschmidt C, van den Bogert AJ, Lundberg A, Nigg BM, Murphy N, Stacoff $A$, et al. Tibiofemoral and tibiocalcaneal motion during walking: external vs. skeletal markers. Gait Posture 1997:6:98-109.

[2] Dennis DA, Mahfouz MR, Komistek RD, Hoff W. In vivo determination of normal and anterior cruciate ligament-deficient knee kinematics. J Biomech 2005;38:241-53.

[3] Mozes A, Chang TC, Arata L, Zhao W. Three-dimensional A-mode ultrasound calibration and registration for robotic orthopaedic knee surgery. Int J Med Robot + Comput Assist Surg: MRCAS 2010;6:91-101.

[4] Banks SA, Hodge WA. Accurate measurement of three-dimensional knee replacement kinematics using single-plane fluoroscopy. IEEE Trans Biomed Eng 1996;43:638-49.

[5] Lin C-C, Lu T-W, Lu H-L, Kuo M-Y, Hsu H-C. Effects of soft tissue artifacts on differentiating kinematic differences between natural and replaced knee joints during functional activity. Gait Posture 2016;46:154-60.

[6] Pandy MG. Computer modeling and simulation of human movement. Annu Rev Biomed Eng 2001;3:245-73.

[7] Akbarshahi M, Schache AG, Fernandez JW, Baker R, Banks S, Pandy MG. Non-invasive assessment of soft-tissue artifact and its effect on knee joint kinematics during functional activity. J Biomech 2010;43:1292-301.

[8] Cappozzo A, Della Croce U, Leardini A, Chiari L. Human movement analysis using stereophotogrammetry: part 1: theoretical background. Gait Posture 2005;21:186-96.

[9] Lu TW, O'Connor JJ. Bone position estimation from skin marker co-ordinates using global optimisation with joint constraints. J Biomech 1999;32:129-34.

[10] Ramsey DK, Wretenberg PF. Biomechanics of the knee: methodological considerations in the in vivo kinematic analysis of the tibiofemoral and patellofemoral joint. Clin Biomech 1999:14:595-611.

[11] Benoit DL, Ramsey DK, Lamontagne M, Xu L, Wretenberg P, Renström P. Effect of skin movement artifact on knee kinematics during gait and cutting motions measured in vivo. Gait Posture 2006:24:152-64.

[12] Leardini A, Chiari L, Croce UD, Cappozzo A. Human movement analysis using stereophotogrammetry: Part 3. Soft tissue artifact assessment and compensation. Gait Posture 2005;21:212-25.

[13] Peters A, Galna B, Sangeux M, Morris M, Baker R. Quantification of soft tissue artifact in lower limb human motion analysis: a systematic review. Gait Posture 2010;31:1-8.

[14] Barr A, Thiran JP, Jolles BM, Theumann N, Aminian K. Soft tissue artifact assessment during treadmill walking in subjects with total knee arthroplasty. IEEE Trans Biomed Eng 2013;60:3131-40.

[15] Garling EH, Kaptein BL, Mertens B, Barendregt W, Veeger HE, Nelissen RG, et al. Soft-tissue artefact assessment during step-up using fluoroscopy and skin-mounted markers. J Biomech 2007;40(Suppl 1):S18-24.

[16] Andersen MS, Benoit DL, Damsgaard M, Ramsey DK, Rasmussen J. Do kinematic models reduce the effects of soft tissue artefacts in skin marker-based motion analysis? An in vivo study of knee kinematics. J Biomech 2010;43:268-73.

[17] Benoit DL, Damsgaard M, Andersen MS. Surface marker cluster translation, rotation, scaling and deformation: their contribution to soft tissue artefact and impact on knee joint kinematics. J Biomech 2015;48:2124-9.

[18] Camomilla V, Donati M, Stagni R, Cappozzo A. Non-invasive assessment of superficial soft tissue local displacements during movement: a feasibility study. J Biomech 2009;42:931-7.

[19] Clément J, Dumas R, Hagemeister N, de Guise JA. Soft tissue artifact compensation in knee kinematics by multi-body optimization: performance of subject-specific knee joint models. J Biomech 2015;48:3796-802.

[20] Freeman MAR, Pinskerova V. The movement of the normal tibio-femoral joint. J Biomech 2005;38:197-208.

[21] Miranda DL, Rainbow MJ, Crisco JJ, Fleming BC. Kinematic differences between optical motion capture and biplanar videoradiography during a jump-cut maneuver. J Biomech 2013;46:567-73.

[22] Andriacchi TP, Alexander EJ, Toney MK, Dyrby C, Sum J. A point cluster method for in vivo motion analysis: applied to a study of knee kinematics. J Biomech Eng 1998;120:743-9.

[23] Garling EH, Kaptein BL, Geleijns K, Nelissen RGHH, Valstar ER. Marker configuration model-based roentgen fluoroscopic analysis. J Biomech 2005;38:893-901.

[24] Anderst W, Zauel R, Bishop J, Demps E, Tashman S. Validation of three-dimensional model-based tibio-femoral tracking during running. Med Eng Phys 2009;31:10-16.

[25] You BM, Siy P, Anderst W, Tashman S. In vivo measurement of 3-D skeletal kinematics from sequences of biplane radiographs: application to knee kinematics. IEEE Trans Med Imaging 2001;20:514-25.

[26] Guan S, Gray HA, Keynejad F, Pandy MG. Mobile biplane x-ray imaging system for measuring 3D dynamic joint motion during overground gait. IEEE Trans Med Imaging 2016;35:326-36.

[27] Barratt DC, Chan CSK, Edwards PJ, Penney GP, Slomczykowski M, Carter TJ et al. Instantiation and registration of statistical shape models of the femur and pelvis using 3D ultrasound imaging. Med Image Anal 2008;12:358-74.

[28] Masum MA, Pickering M, Lambert A, Scarvell J, Smith P. Accuracy assessment of Tri-plane B-mode ultrasound for non-invasive 3D kinematic analysis of knee joints. Biomed Eng Online 2014;13:122. 
[29] Hamidzada WA, Osuobeni EP. Agreement between A-mode and B-mode ultrasonography in the measurement of ocular distances. Vet Radiol Ultrasound: Off J Am Coll Vet Radiol Int Vet Radiol Assoc 1999;40:502-7.

[30] Masum MA, Pickering MR, Lambert AJ, Scarvell JN, Smith PN. Precision analysis of single-element ultrasound sensor for Kinematic analysis of knee joints. Electron Lett 2014;50:1047-8.

[31] Miranda DL, Rainbow MJ, Leventhal EL, Crisco JJ, Fleming BC. Automatic determination of anatomical coordinate systems for three-dimensional bone models of the isolated human knee. J Biomech 2010;43:1623-6.

[32] Inc. PPT. VZ4000v technical specifications.

[33] Azhari H. Appendix A: typical acoustic properties of tissues. Basics of biomedical ultrasound for engineers. John Wiley \& Sons, Inc; 2010. p. 313-14.

[34] Barratt DC, Penney GP, Chan CS, Slomczykowski M, Carter TJ, Edwards PJ, et al. Self-calibrating 3D-ultrasound-based bone registration for minimally invasive orthopedic surgery. IEEE Trans Med Imaging 2006;25:312-23.

[35] Grood ES, Suntay WJ. A joint coordinate system for the clinical description of three-dimensional motions: application to the knee. J Biomech Eng 1983;105:136-44.
[36] Horn BKP. Closed-form solution of absolute orientation using unit quaternions. J Opt Soc Am A 1987;4:629-42.

[37] Cappozzo A, Catani F, Leardini A, Benedetti MG, Della Croce U. Position and orientation in space of bones during movement: experimental artefacts. Clin Biomech 1996;11:90-100.

[38] Cappozzo A, Catani F, Croce UD, Leardini A. Position and orientation in space of bones during movement: anatomical frame definition and determination. Clin Biomech (Bristol, Avon) 1995;10:171-8.

[39] Bingham J, Li G. An optimized image matching method for determining in-vivo TKA kinematics with a dual-orthogonal fluoroscopic imaging system. J Biomech Eng 2006;128:588-95.

[40] Giphart JE, Zirker CA, Myers CA, Pennington WW, LaPrade RF. Accuracy of a contour-based biplane fluoroscopy technique for tracking knee joint kinematics of different speeds. J Biomech 2012;45:2935-8. 